\title{
Technique of Splenopexy for Wandering Spleen
}

\author{
Lauren McCormack, MD, Wilbur Bowne, MD \\ Department of Surgery, Drexel University College of Medicine, Philadelphia, Pennsylvania, USA \\ (Drs McCormack and Bowne).
}

\begin{abstract}
Introduction: Wandering spleen is a rare condition in which the spleen lacks or has extreme laxity of associated ligaments, enabling it to float freely throughout the abdomen. This condition most commonly presents as splenic ischemia secondary to torsion of the vascular pedicle and is most commonly treated with splenectomy over splenic salvage.

Case Description: We present the case of a 34-year-old woman with wandering spleen who underwent a technique for laparoscopic splenopexy in which a fibrin sealant is applied between the splenic capsule and lateral abdominal wall for splenic fixation.
\end{abstract}

Discussion: This technique can be performed by surgeons trained in basic laparoscopy and may be used to increase splenic salvage.

Key Words: Splenopexy, Wandering spleen.

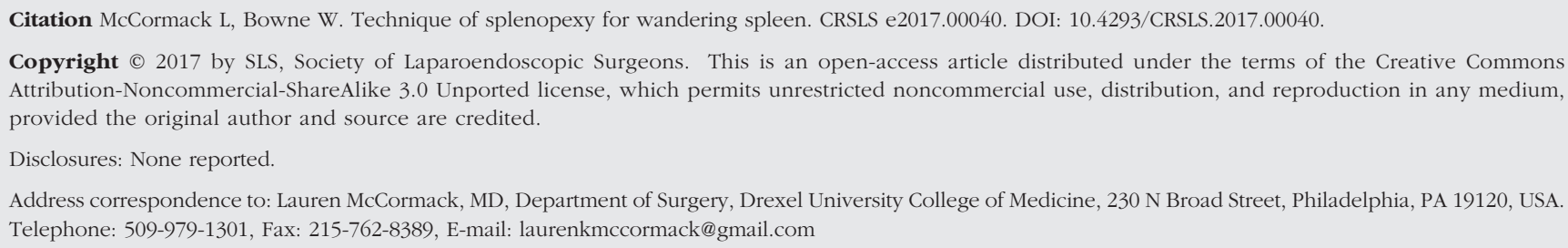

\section{INTRODUCTION}

Wandering spleen is a rare disorder that allows the spleen to roam unhindered throughout the abdomen, due to deficiency or laxity of the splenic ligaments. It was first described during an autopsy in 1667 , but the true prevalence remains unknown, as most patients are asymptomatic. ${ }^{1}$ This condition most commonly presents as splenic ischemia secondary to torsion of the vascular pedicle. ${ }^{2}$ Thirty percent of cases are seen in children younger than 10 years, where the condition is congenital. In adults, it is more commonly an acquired condition. Women are 10 times more likely to be affected than are men, with the condition usually occurring in multiparous women between ages 20 and 40. It is hypothesized that excess estrogen of pregnancy causes laxity and eventual tearing of the splenic ligaments. ${ }^{2}$

Wandering spleen accounts for less than $0.25 \%$ of splenectomies. ${ }^{3}$ Splenectomy is the most common treatment compared with splenic salvage procedures. The risk of overwhelming postsplenectomy infection (OPSI) is $0.13-8.1 \%$, carrying a mortality of $30 \%$ to $60 \%$, illustrating that splenic salvage is preferable. ${ }^{4}$ Although splenic salvage is not always possible, there is an increased trend toward splenopexy, as splenectomy rates have decreased from $98 \%$ to $52 \%$ for this condition. ${ }^{2}$

A variety of open and laparoscopic techniques for splenopexy are described. ${ }^{4}$ Laparoscopic techniques mirror the same principals as open techniques, but laparoscopy has the advantage of less postoperative pain and disability plus decreased length of stay (LOS). The most commonly described technique involves fashioning a Vicryl or Dexon mesh bag around the spleen and securing it to the lateral abdominal wall, followed by an omentopexy around the spleen, which can be fixed to the abdominal wall or diaphragm. ${ }^{4}$ Another technique creates a peritoneal pocket along the lateral abdominal wall. ${ }^{2,4}$ The spleen is subsequently placed within this pocket in the preperitoneal space, sutured closed around the splenic hilum, and secured by omentopexy. We report a technically simpler method of treating wandering spleen. 

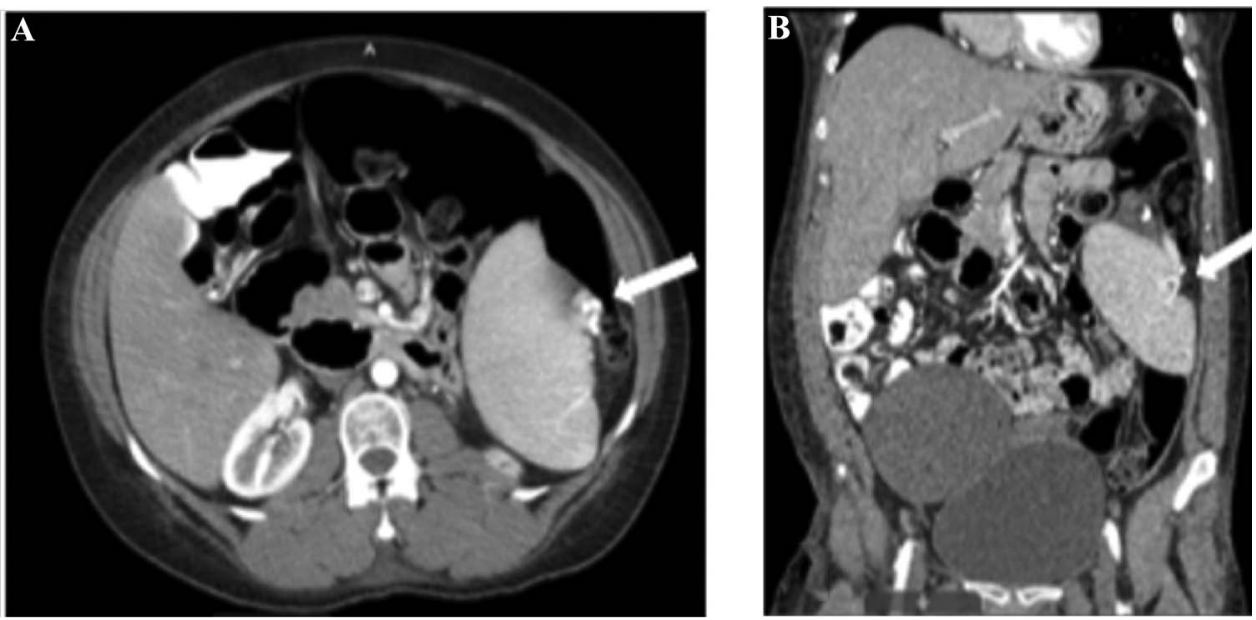

Figure 1. (A) CT axial image with volvulus of the spleen with the splenic hilum pointing lateral (arrow). (B) CT coronal with volvulated spleen seen in the mid abdomen (arrow) and an incidentally discovered adnexal mass in the right lower quadrant.

\section{CASE REPORT}

A 34-year-old female with a medical history of hepatitis C and heroine abuse presented to the emergency department with complaints of worsening chronic upper abdominal pain over the course of several months. Abdominal CT demonstrated an inverted splenic hilum and volvulus (Figure 1) with a whirling of the splenic vascular pedicle and distal pancreas (Figure 2). An incidentally discovered adnexal mass was also noted. Her abdominal pain was in the left upper quadrant, intermittent and crampy in nature, consistent with chronic, intermittent

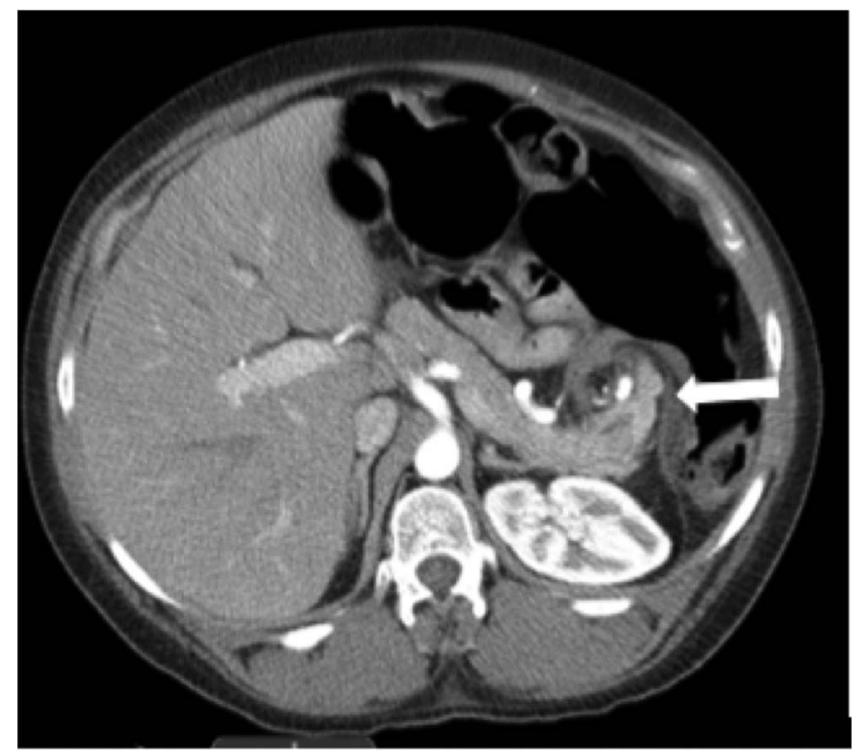

Figure 2. CT axial image demonstrating the distal pancreatic swirl (arrow) with splenic vascular pedicle. torsion of the spleen. A joint procedure was planned with the gynecologic service for splenopexy and removal of the adnexal mass.

\section{Technique}

For entry into the abdomen and dual procedural purposes, we used a 4-trocar approach. We placed a Hasson trocar at the umbilicus and used a 10-mm, 30-degree laparoscope. A 10-mm working port was placed in the right upper quadrant adjacent to the falciform ligament, to accommodate the laparoscopic argon beam. Working and assistant $5-\mathrm{mm}$ ports were placed $10 \mathrm{~cm}$ right-lateral and left-lateral to the Hasson port, respectively (Figure 3). The spleen was manipulated and found to be completely without peritoneal attachments and moving freely around its vascular pedicle. When we evaluated the splenic vascular pedicle and distal pancreas, we found them to be in normal anatomic position without torsion of the redundant vessels.

The goal of our procedure was to encourage adhesion formation between the splenic capsule and peritoneum of the adjacent lateral abdominal wall. The splenic capsule on the convex surface of the spleen was thoroughly scored with an argon beam coagulator. Next, the peritoneal surface of the lateral abdominal wall was similarly scored. The spleen was then situated laterally in the normal anatomic position to assure that the scored surfaces were in contact, while ensuring that the vasculature was not rotated. Fibrin sealant (human) (Evicel; Ethicon, Somerville, New Jersey) was then placed between the convex surface of the spleen and the lateral abdominal 


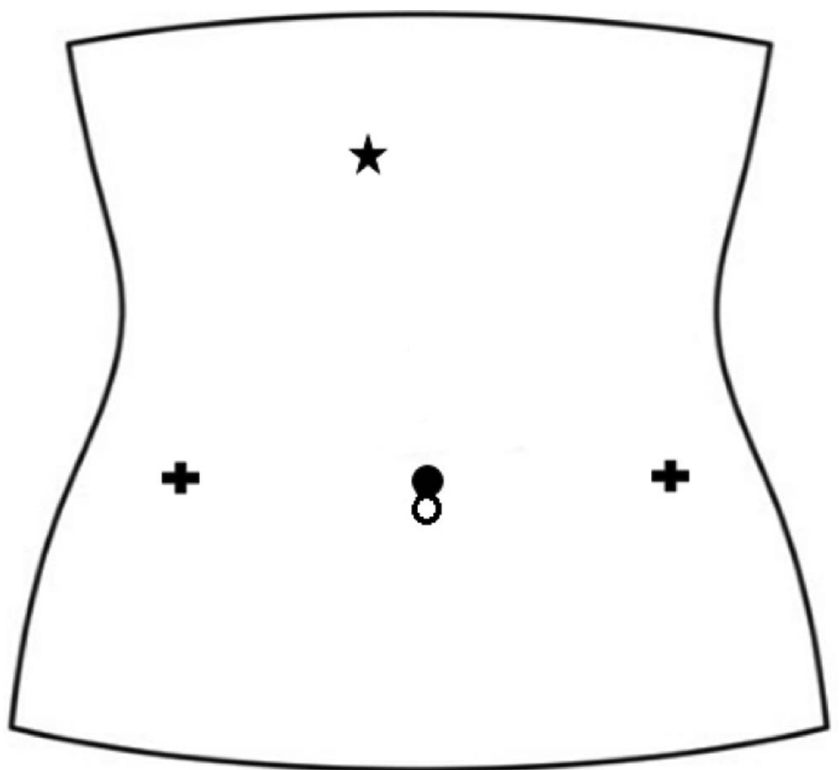

Figure 3. Illustration of 4-port placement. Circle: Hasson trocar. Star: 10-mm port to accommodate Argon beam. Crosses: 5-mm ports, left lower quadrant is the working port and right lower quadrant port is the assistant port. The technique is amenable to 3-port placement.

wall peritoneum to secure the spleen. A flexible, extended tip was used for the fibrin sealant and guided with a laparoscopic grasper (Figure 4). Lastly, redundant gastrocolic ligament was tacked around the spleen creating a layer of anterior and medial support (Figure 5). Alternatively, the omentum can be used for fixation if the gastrocolic ligament is deemed unsuitable. At the end of the procedure, the spleen was securely attached to the left upper quadrant. Our portion of the procedure took approximately 30 minutes. The patient remained in a nonbiased supine position throughout the procedure.
After surgery the patient did well, tolerated her diet, and was discharged home. Five months after surgery the patient's abdominal pain had resolved, and she reported improvement in the chronic constipation. Postoperative computed tomographic scans at 1 week and 5 months demonstrated the spleen in the left upper quadrant, without twisting of the vascular pedicle surrounded by tacks from the omentopexy (Figure 6). Remarkably, the spleen's position within the left upper quadrant now shows respiratory-dependent positional variation, as the spleen appears adherent to the diaphragmatic parietal peritoneum and underlying musculature.

\section{DISCUSSION}

Our technique provides a splenopexy option that is safe and time efficient, without technical difficulties. As previously described, creation of a preperitoneal pocket and atraumatic manipulation and positioning of the spleen within it can be technically challenging. Hand-assisted ports can be helpful, but require larger incisions with increased rates of hernia formation and postoperative pain. There also remains an inherent risk of vascular torsion or tension on the splenic hilum with a peritoneal pocket technique. A Vicryl mesh bag, or any mesh, can create peritoneal adhesions and eventual bowel obstruction. ${ }^{5}$

The technique's concept is borrowed from Pleurodesis. The Argon beam will superficially coagulate the convex surface of the spleen, care must be taken to avoid scoring the hilum or short gastrics. Any portion of the convex surface of the spleen that will have contact with the abdominal wall should be treated with energy. A superficial and thorough scoring of the splenic capsule and lateral abdominal wall peritoneum creates the raw surface
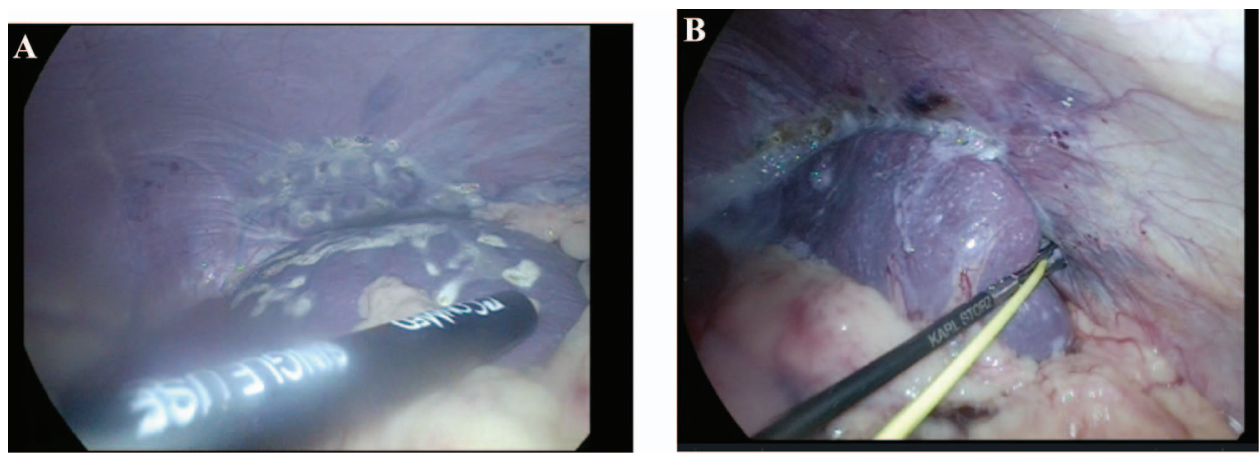

Figure 4. (A) Scoring the peritoneum and splenic capsule with laparoscopic Argon beam. (B) Laparoscopic extension of Evicel kit and grasper to guide sealant between the splenic capsule and the lateral abdominal wall. 

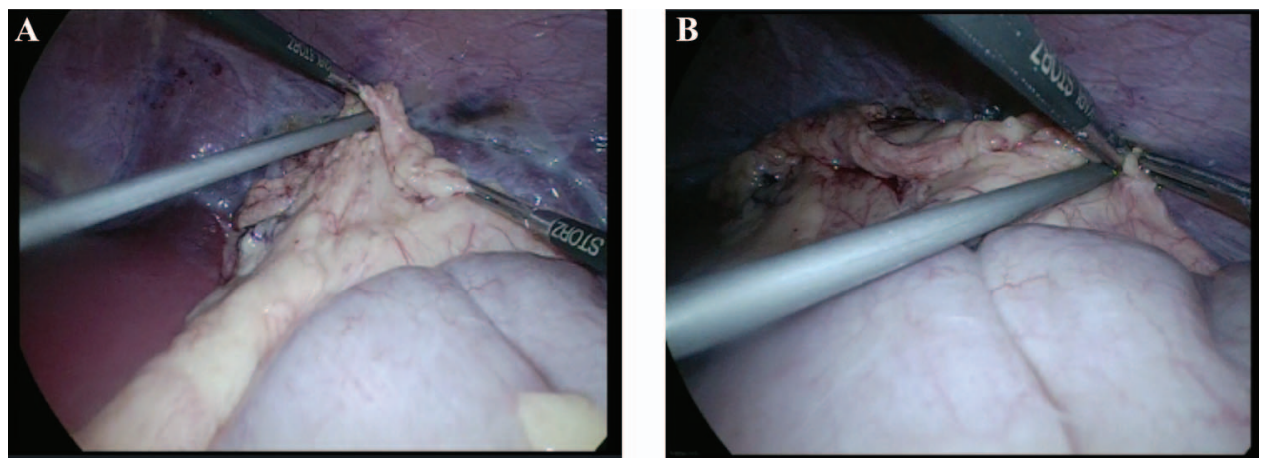

Figure 5. (A) Fixation of gastrocolic ligament with 5-mm tacks for anterior and medial support. (B) The gastrocolic ligament was secured anterior and inferior, to create a pocket surrounding the spleen.
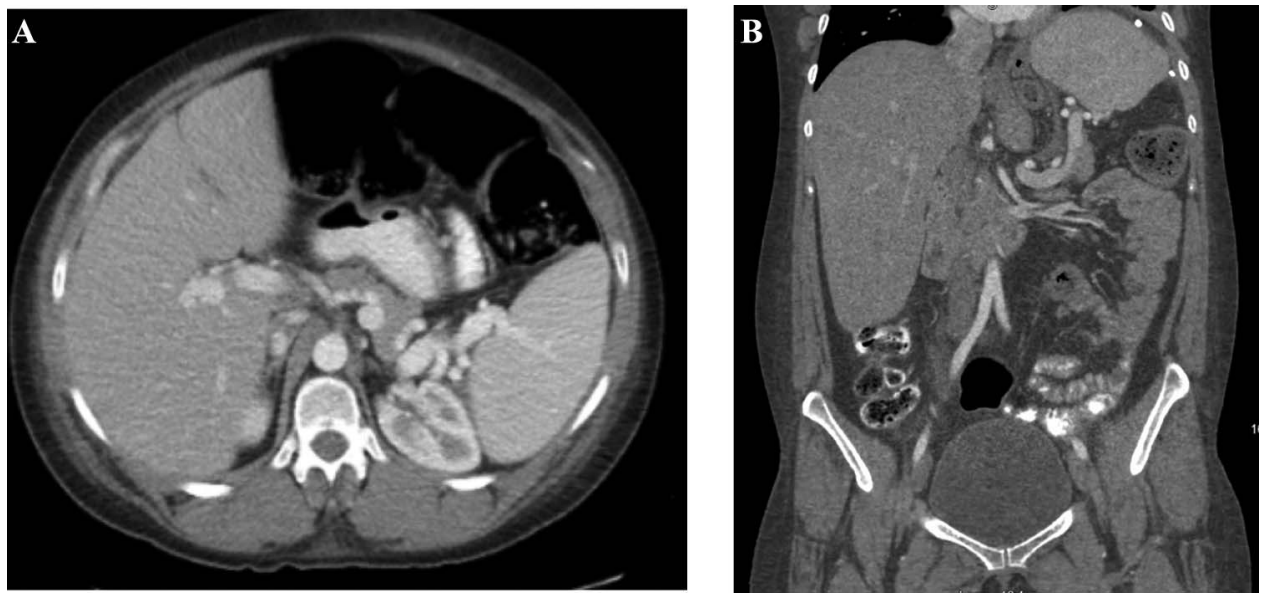

Figure 6. (A) CT axial image. Postoperative demonstration of anatomically adherent/fixed spleen with nontorsed vascular pedicle in left upper quadrant (week 1). (B) CT coronal image. Postoperative demonstration of spleen with nontorsed vascular pedicle in left upper quadrant with tacks seen in the diaphragmatic parietal peritoneum and underlying musculature demonstrating respiratory dependent positional variation (5 months).

needed for adhesion formation and provides hemostasis. The Argon beam is less likely to cause deeper injury compared to Bovie cautery or Harmonic scalpel. In addition, most institutions have access to the Argon beam, while other newer energy technologies are unavailable outside larger centers.

Current adhesive technology can assist in avoiding placement of mesh in the abdomen where it can have direct contact with bowel. The use of fibrin adhesive is well described in the hernia literature as a method of mesh fixation. 6,7 In the hernia literature, the fibrin sealant is used to create a temporary adherence of the spleen and the abdominal wall. This allows stronger attachments to mature from the Argon beam treatment. No bleeding was encountered during treatment with Argon beam, but the fibrin sealant has the additional advantage of being a hemostatic agent. ${ }^{6,7}$

This technique can be extrapolated for use in solid organopexy. Our technique can be performed by surgeons trained in basic laparoscopy, and a consideration for splenopexy/splenic salvage instead of splenectomy. It is safe and efficient, limiting use of operating room and anesthesia resources to a minimum.

\section{CONCLUSION}

This report describes a minimally invasive technique for splenopexy that, to our knowledge, is not described in the literature. It is an alternative, effective and nonchallenging technique that a general surgeon with basic laparoscopic skills can perform. In general, it uses adhesions, fibrin 
adhesive and omental buttressing to secure the wandering spleen.

\section{References:}

1. Karaosmanoglu AD, Onur MR, Karcaaltincaba M. Wandering spleen with volvulus of pancreas. J Med Ultrasonics. 2015;42: 413-416.

2. Soleimani M, Mehrabi A, Kashfi A, Fonouni H, Buchler MW, Kraus TW. Surgical treatment of patients with wandering spleen: report of six cases with a review of the literature. Surg Today. 2007;37:261-269.

3. Flores-Rios E, Mendez-Diaz C, Rodrirquez-Garcia E, PerezRamos T. Wandering spleen, gastric and pancreatic volvulus and right-sided descending and sigmoid colon. Gastrointest Radiol. 2015;9:18-25.
4. Fiquet-Francois C, Belouadah M, Ludot $\mathrm{H}$, et al. Wandering spleen in children: multicenter retrospective study. J Pediatr Surg. 2010;45:1519-1524.

5. Sloan DA. Complete small bowel obstruction in the early postoperative period complicating surgical sling procedure. Am Surg. 1994;60:282-286.

6. Berney CR, Descallar J. Review of 1000 fibrin glue mesh fixation during endoscopic totally extraperitoneal (TEP) inguinal hernia repair. Surg Endosc. 2016;30:4544-4552.

7. Sajid MS, Ladwa N, Kalra L, McFall M, Baig MK, Sains P. A meta-analysis examining the use of tacker mesh fixation versus glue mesh fixation in laparoscopic inguinal hernia repair. Am J Surg. 2013;206:103-111. 